Journal of

Synchrotron

Radiation

ISSN 0909-0495

Received 21 July 2009

Accepted 7 December 2009

\section{Development of a differential pumping system for soft X-ray beamlines for windowless experiments under normal atmospheric conditions}

\author{
Y. Tamenori
}

Japan Synchrotron Radiation Research Institute/SPring-8, 1-1-1 Kouto, Sayo, Hyogo 679-5198, Japan. E-mail: tamenori@spring8.or.jp

\begin{abstract}
A novel design for a differential pumping system has been investigated. This system allows windowless experiments in a soft X-ray beamline under normal atmospheric conditions. The new design consists of an aperture-based four-stage differential pumping system, based on a simple model calculation. A prototype system with a total length of $600 \mathrm{~mm}$ was constructed to confirm the validity of the design concept. Relatively short conductance-limiting components allow easy installation and alignment of the system on a synchrotron beamline. The fabricated system was installed on a beamline to test the transmission of soft $\mathrm{X}$-rays through atmospheric helium.
\end{abstract}

Keywords: vacuum; differential pump; windowless connection; helium path; soft X-rays.

\section{Introduction}

In synchrotron radiation facilities, thin metal and polymer films are usually used as a vacuum window to separate the ultra-high vacuum of the storage ring or the optics chamber from the high-pressure environment of the experimental apparatus. A beryllium window is often used as a standard front-end component owing to its high optical transmission of the X-ray beam. However, Be windows absorb lower-energy photons, and cannot be used for soft X-ray beamlines. Instead, the standard procedure in a soft X-ray beamline is to install all equipment necessary for experiments inside the high-vacuum chamber. Recently, demands to study samples under higher pressure (1-760 mbar) have increased in the soft X-ray region, which are in some cases quite different from those in a highvacuum condition (Pantförder et al., 2005).

Recently, several groups have attempted to obtain photoemission data at pressures of up to 100 mbar by separating the sample region from the beamline using a vacuum window (Ogletree et al., 2002; Pantförder et al., 2005). The vacuum windows used in the soft X-ray regions need to be ultra-thin in order to transmit the photons to the sample region. However, thin metal films are fragile, and have difficulty withstanding a high pressure difference. Furthermore, organic contamination on the vacuum window reduces the photon intensity in the absorption edges of light elements. In order to overcome these complications, the development of a windowless method must be investigated. The development of a windowless connection between a high-vacuum beamline and a high-pressure sample chamber would provide an opportunity to open new scientific possibilities in soft X-ray sciences.
Hard X-ray experiments could also benefit from a windowless connection, though Be windows are usually used in the hard X-ray region. Since they are not always optically flat on the X-ray wavelength scale, the windows cause a degradation of the beam quality, which would be an especially serious problem when using coherent X-ray beams (Gog et al., 2007). New developments in X-ray sources have provided $\mathrm{X}$-ray beams of higher coherency, which has lead to new X-ray analytical techniques that were not feasible just a decade ago. Elimination of vacuum windows from the beamline also creates the possibility to improve the X-ray quality in the hard $\mathrm{X}$-ray region.

Recently, increased effort has been expended in the development of optically high-quality vacuum windows (Goto et al., 2004; Blumer et al., 2006). Another approach to overcoming the window problem, which might be a simpler solution, is to use a differential pumping system (Warburton \& Piantetta, 1990). In a differential pump, the conductance between two vacuum chambers is limited by an open aperture or pipes. It allows photons of all energy ranges to pass freely along a line of the light path between the two different pressure regions. The differential pumping system is widely used in ultra-high-vacuum experiments using a molecular beam (Haberland, 1994; Pollard et al., 1981; Ran et al., 2007) and a helium discharge lamp (Rowe et al., 1973, 1985). Furthermore, the differential pump has already been installed on beamlines in many synchrotron radiation facilities, e.g. in place of the Be window (Renier \& Draperi, 1997), as a gas filter (Lee et al., 2001; Mercier et al., 2000) and in CVD reaction chambers (Ohashi et al., 2001a). Advances in vacuum technology and the brilliant photon beam produced by a modern synchrotron 
light source enable us to eliminate vacuum windows from the beamline. Recently, a windowless connection was achieved between a high-vacuum chamber and an atmospheric pressure environment using a differential pump with millimeter-sized beam passes (Gog et al., 2007).

In the present study, a differential pumping system was designed for use with windowless soft X-ray experiments under normal atmospheric conditions. The assembly prototype was fabricated to confirm the feasibility of the design concept, and was installed on the soft X-ray beamline at SPring-8 (BL27SU). This differential pumping system creates a windowless connection between the high-vacuum chamber and a sample region with an atmospheric pressure environment. It was a challenge to create a windowless connection between an experimental chamber filled by $1 \mathrm{~atm}$ helium and the high-vacuum beamline. Since soft X-rays at around $1.0 \mathrm{keV}$ are fairly absorbed in atmospheric air, a He path was used instead of an air path (Roper et al., 1992; Yagi et al., 2004). While He gas has a higher transmission than air in the soft X-ray region owing to its smaller atomic number, it is more difficult to evacuate $\mathrm{He}$ using vacuum pumps. The performance of the differential pump was evaluated by connecting it to both atmospheric air and helium environments. The transmission of soft X-rays was measured under the condition of atmospheric helium to demonstrate the viability of the apparatus.

\section{Considerations for the system design}

The performance of the differential pump strongly depends on the aperture size and the length of the conductance-limiting components. When the size of the aperture is reduced, or the length of the pipe is elongated, the gas flow is decreased. However, the photon beam has to pass through the conductance-limiting components. Narrower and longer pipes are inconvenient for these experiments, even though recent synchrotron radiation light sources produce a very narrow photon beam. When designing a new differential pump, it is important to consider both the pump's performance and the convenience of apparatus. From this point of view, an aperture-based differential pump is more attractive when attempting to construct a compact system, although the performance of a differential pump is less effective than that of a system constructed using a long pipe.

Gas flow in a vacuum system has been extensively investigated. The vacuum theory has been described in detail in literature on vacuum technology (Rozanov \& Hablanian, 2002; O'Hanlon, 2003). In the present report, we do not go into the details of vacuum theory and technology, but only present the formula used for our simulation. Fig. 1 illustrates the model system of a proposed differential pump. $P_{0}$ and $P_{1}$ denote the pressure of the higher and lower pressure regions $\left(P_{0}>P_{1}\right)$, respectively. The $P_{1}$ region is evacuated by a vacuum pump with a pumping speed of $S_{1}\left(\mathrm{~L} \mathrm{~s}^{-1}\right)$. The two regions are connected by a cylindrical conductance-limiting component of diameter $D(\mathrm{~cm})$ and length $L(\mathrm{~cm})$. The gas flow $Q$ through

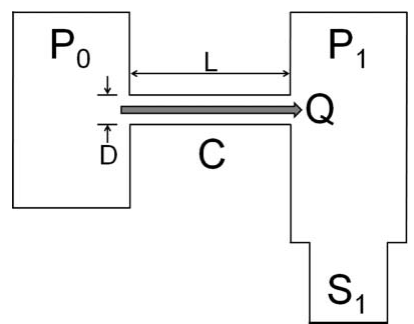

Figure 1

Schematic diagram of the model system of the differential pump. $P_{0}$ and $P_{1}$ denote the pressure of higher and lower pressure regions, respectively. $S_{1}$ is the pumping speed, $Q$ is the gas flow, $C$ is the conductance and $D$ and $L$ are the diameter and length of the conductance-limiting component, respectively.

the conductance-limiting component is given by the following equation,

$$
Q=C\left(P_{0}-P_{1}\right) \quad\left(\mathrm{Pa} \mathrm{L} \mathrm{s}{ }^{-1}\right),
$$

where $C$ is the conductance of the system. In an actual differential pumping system, an ideal thin aperture cannot be used because it would not withstand the pressure difference. Therefore, the formulation of a thick aperture, i.e. short tube, is significant for our purpose. In the present study, the models proposed by Santeler were used in the optimization of the system (Santeler, 1986a,b, 1994). Santeler formulated the treatment of a short round tube in all gas-flow regions (molecular, viscous and intermediate). Santeler's model treats the tube as an aperture in a series with a tube of length $L$. This treatment allows us to separate the exit conductance from the tube conductance, and the exit effect, when the pressure drops at the tube exit, can be accounted for in the estimation.

In the present system, the gas flow is treated as a choked flow. The limit of choked flow can be determined by the following equation,

$$
\frac{P_{1}}{P_{0}} \leq\left(\frac{2}{\gamma+1}\right)^{1 /(\gamma-1)}
$$

where $\gamma$ is the specific heat ratio. For air and helium, $\gamma$ is 1.40 and 1.67 , respectively. Hence, the $P_{1} / P_{0}$ ratio is $\sim 0.5$. The present differential pumping system easily satisfies this condition, because it only requires the downstream pressure to be below one-half of the upstream pressure.

For a molecular flow region, when the mean free path of the gaseous molecule is longer than the diameter of the aperture, the pioneering work to formulate the gas flow in a short tube was carried out by Clausing (1932). He proposed an equation of conductance for the general case where any component serves as a connection between two large volumes,

$$
C_{\mathrm{M}}=\alpha C_{\text {M_orifice }} \quad\left(\mathrm{L} \mathrm{s}^{-1}\right),
$$

where $\alpha$ is the Clausing factor, which is a translation probability that a particle entering the pipe at one end will escape at the other end after making diffuse collisions with the wall of the pipe. Clausing also developed integral equations for the exact solution of $\alpha$ for circular tubes of any length-to-radius ratio. Later, Santeler further developed the Clausing model, 
and proposed a simple formula for calculating the Clausing factor, taking into account the exit effect in a short tube (Santeler, 1986a,b),

$$
\alpha=\frac{1}{1+3 L^{\prime} / 4 D},
$$

where

$$
L^{\prime}=L\left(1+\frac{1}{3+3 L / 3.5 D}\right)
$$

$C_{\mathrm{M} \_ \text {orifice }}$ is the conductance for thin circular orifices, which is given by the following equation,

$$
C_{\text {M_orifice }}=(\pi / 16) \bar{\nu} D^{2}=0.09(T / M)^{1 / 2} D^{2} \quad\left(\mathrm{~L} \mathrm{~s}^{-1}\right),
$$

where $T$ is the Kelvin temperature and $M$ is the mass of gas. The gas flow $Q_{\mathrm{M}}$ can be estimated using following equation,

$$
Q_{\mathrm{M}}=0.09(T / M)^{1 / 2} D^{2} \alpha\left(P_{0}-P_{1}\right) \quad\left(\mathrm{Pa} \mathrm{L} \mathrm{s}^{-1}\right) .
$$

The gas flow $Q_{\mathrm{M}}$ depends on the mass of the gas travelling through. For example, in the present system, air at $T=$ $293.15 \mathrm{~K}$ and $M=0.029 \mathrm{~kg} \mathrm{~mol}^{-1}$ has the following values,

$$
C_{\text {M_Air }}=9.1 \alpha D^{2} \quad\left(\mathrm{~L} \mathrm{~s}^{-1}\right) \text {. }
$$

On the other hand, helium at $T=293.15 \mathrm{~K}$ and $M=$ $0.004 \mathrm{~kg} \mathrm{~mol}^{-1}$ has the following values,

$$
C_{\mathrm{M} \_\mathrm{He}}=24.5 \alpha D^{2} \quad\left(\mathrm{~L} \mathrm{~s}^{-1}\right) \text {. }
$$

When helium is evacuated, the gas flow increases by a factor of about 2.6.

In order to design this differential pumping system, it is necessary to estimate the gas flow in the viscous flow region, where the mean free path of the gaseous molecule is shorter than the diameter of the aperture. The viscous flow for the short pipe $Q_{\mathrm{V}}$ is given by the following equation (Santeler, $1986 b)$,

$$
Q_{\mathrm{V}}=\left(C^{2} / 2 K\right)\left\{\left[1+\left(2 K P_{0} / C\right)^{2}\right]^{1 / 2}-1\right\} \quad\left(\mathrm{Pa} \mathrm{L} \mathrm{s}^{-1}\right),
$$

with

$$
C=24.06 D^{2}\left\{\gamma(T / M)[2 /(\gamma+1)]^{\gamma+1 / \gamma-1}\right\}^{1 / 2}
$$

and

$$
K=\pi D^{4} / 192 \eta L,
$$

where $\eta$ is the coefficient of viscosity. The gas flow predicted by (6) and (7) is plotted in Fig. 2. In the transition region, i.e. the intermediate region between the viscous and the molecular region, the gas flow curves obtained from (6) and (7) are discontinuous. The treatment of gas flow in the transition region for a short tube was proposed by Santeler (1994),

$$
Q_{\mathrm{T}}=\theta Q_{\mathrm{V}}+(1-\theta) Q_{\mathrm{M}} \quad\left(\mathrm{Pa} \mathrm{L} \mathrm{s}^{-1}\right),
$$

with

$$
\theta=R /(\bar{P}+R),
$$

where $R$ is the midpoint pressure of the transition region and $\bar{P}$ is the average pressure. In the present system, $R$ was fixed as

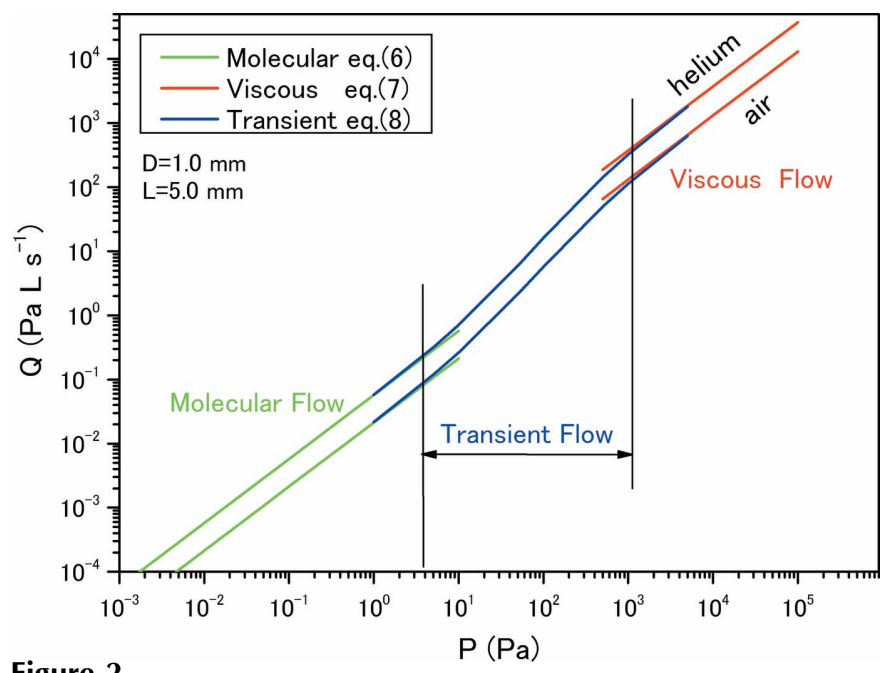

Figure 2

Estimated pressure dependency of gas flow in the molecular, viscous and transition regions.

$65 \mathrm{~Pa}$ (air) and $192 \mathrm{~Pa}$ (helium), thus the Knudsen number becomes 0.1 . Fig. 2 indicates that (8) smoothly connects two flow regions. Equations (6), (7) and (8) were used in the simulations discussed in a later section, as gas flow for the molecular, viscous and transition flow regions, respectively.

\section{Fabrication of a prototype differential pumping system}

A four-stage differential pumping system was fabricated to verify the design concept discussed in the present report. A schematic drawing of the proposed system is shown in Fig. 3. The sizes of apertures and the pumping speeds of the vacuum pumps were optimized using the simulation of a model system. The system consists of two vacuum chambers. The first chamber contains the first two conductance-limiting apertures ( $D_{1}$ and $D_{2}$ ), and serves as the first and the second stages of the differential pump. The second chamber contains the third and fourth apertures $\left(D_{3}\right.$ and $\left.D_{4}\right)$, and serves as the second, third and fourth stages of the differential pump. Both cham-

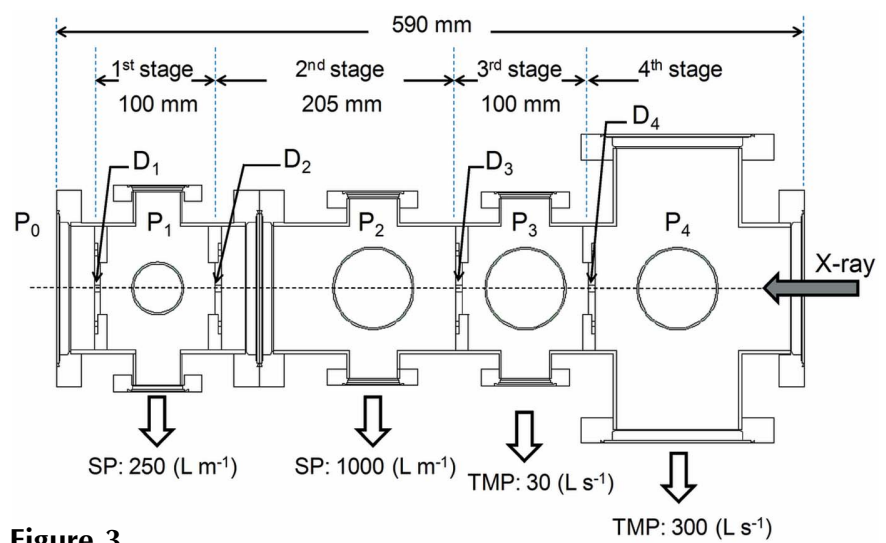

Figure 3

TMP: $300\left(\mathrm{~L} \mathrm{~s}^{-1}\right)$

Schematic drawing of the prototype differential pumping system. $D_{x}(x=$ $1-4)$ is the diameter of each conductance-limiting aperture, and $P_{x}(x=$ $0-4$ ) is the pressure at each differential pumping stage. The arrows at the upper side indicate the length of the system. SP stands for scroll pump and TMP for turbomolecular pump. 
bers were fabricated using a $96.0 \mathrm{~mm}$-diameter SS304 stainless steel pipe, and joined using ICF-152 vacuum flanges.

The gas pressure in the first stage is in a viscous flow region, whereas that in the second stage is in a transition flow region. The role of these two stages is to reduce the pressure of the gas, so as to use a turbomolecular pump in the third stage. The diameter of $D_{1}$ is $1.0 \mathrm{~mm}$. $D_{1}$ is placed between the first differential pumping stage and the sample region of the atmospheric environment $\left(P_{0}\right)$. The first stage is $100.0 \mathrm{~mm}$ long, evacuated using an oil-free scroll vacuum pump (ANEST IWATA ISP-250) with a nominal pumping speed of $250 \mathrm{~L} \mathrm{~min}^{-1}$. The vacuum pump must constantly evacuate the high-pressure chamber. If an oil-sealed vacuum pump were used, serious oil contamination occurs inside the chambers. Thus, we selected oil-free vacuum pumps. $D_{2}$ also has a diameter of $1.0 \mathrm{~mm}$, and was installed inside the first chamber. The second stage is $205.0 \mathrm{~mm}$ long and extends over the two chambers, evacuated using a larger oil-free scroll vacuum pump (ANEST IWATA ISP-1000) with a nominal pumping speed of $1000 \mathrm{~L} \mathrm{~min}^{-1}$.

The diameters of $D_{3}$ and $D_{4}$ are $1.0 \mathrm{~mm}$ and $2.0 \mathrm{~mm}$, respectively. Both apertures are mounted in the second chamber. The third stage is $100.0 \mathrm{~mm}$ long and is evacuated using a hybrid turbomolecular pump (ADIXEN ATH31+) with a nominal pumping speed of $30 \mathrm{~L} \mathrm{~s}^{-1}$. The fourth stage is evacuated using a turbomolecular pump (EDWARDS iXP450 ) with a nominal pumping speed of $300 \mathrm{~L} \mathrm{~s}^{-1}$. Since the gas pressure in the third and fourth stages is in a molecular flow region, it was necessary to mount the vacuum pumps directly on the vacuum chamber to obtain the maximum effective pumping speed. Both turbomolecular pumps were directly mounted on the top or side flanges of the chamber without using the inlet mesh.

The maximum pumping speed of the turbomolecular pump at the third stage is only $30 \mathrm{~L} \mathrm{~s}^{-1}$, which appears to be insufficient for the differential pump. However, in the molecular flow region, the effective pumping speed strongly depends on the conductance between the chamber and the vacuum pump. We can obtain a sufficient pumping speed by mounting the small turbomolecular pump directly on the vacuum chamber even if the pumping speed of a pump is small. The validity of this concept was confirmed by the simulation and the test measurements using the prototype system.

The conductance-limiting apertures $D_{1}-D_{4}$ were installed from both ends of the chamber and rigidly mounted on aperture housing plates welded inside the chambers. For the beam alignment, the plates were adjusted to be centered in the chambers and coaxially aligned along the beam axis. This method mechanically guarantees the precise alignment of the four apertures with accuracy below $0.1 \mathrm{~mm}$. The conductancelimiting apertures were made of SS304 stainless steel plates with a thickness of $5.0 \mathrm{~mm}$ for sufficient rigidity.

Three capacitance vacuum gauges, appropriate for the different pressure regions $\left(P_{1}-P_{3}\right)$, were used to measure the pressures of the first three stages (CANON ANELVA; M-340DG-13, M-340DG-11 and M-340DG-QA). A coldcathode gauge (PFIFFER vacuum; IKR050) was used for the measurement of the vacuum at the fourth stage $\left(P_{4}\right)$. The sensitivity of a cold-cathode gauge for helium gas is greatly different from that for air. The sensitivity was corrected by using the relationship between the actual pressure and the reading from the pressure gauge, as described in the operation manual provided by the manufacturer; $P_{\mathrm{He}}($ actual $)=5.9 P_{\mathrm{He}}$ (gauge reading).

The scale of the system is also indicated in Fig. 3. The total length of the differential pumping system, including all of the components, is less than $600 \mathrm{~mm}$. This length is significantly shorter than other similar systems that have been reported (Gog et al., 2007). The $600 \mathrm{~mm}$ size is of essential importance to SPring- 8 , since the size of the standard pumping unit installed in SPring-8 is of a similar size (Ohashi et al., 2001b). The proposed system can replace the standard pumping unit currently installed on the beamlines of SPring- 8 .

\section{Performance tests}

\subsection{Differential pumping tests}

Fig. 4 shows the pressure distribution curves obtained from the simulation and the test measurement of the prototype. The pressure $P_{0}$ in the sample region is $1 \times 10^{5} \mathrm{~Pa}(=1 \mathrm{~atm})$. The conductance used in the simulation was obtained from equations (6)-(8). The pumping speeds used in the simulation were pressure-dependent values, and they are indicated on the right-hand side of Fig. 4. The pumping speed characteristics for all vacuum pumps were supplied by their respective manufacturers. The pumping speed of the scroll pumps for air and helium is almost the same. The first chamber and the scroll pumps were connected by a $1 \mathrm{~m}$-long flexible tube. The pumping speed curves shown in Fig. 4 are modified, consid-

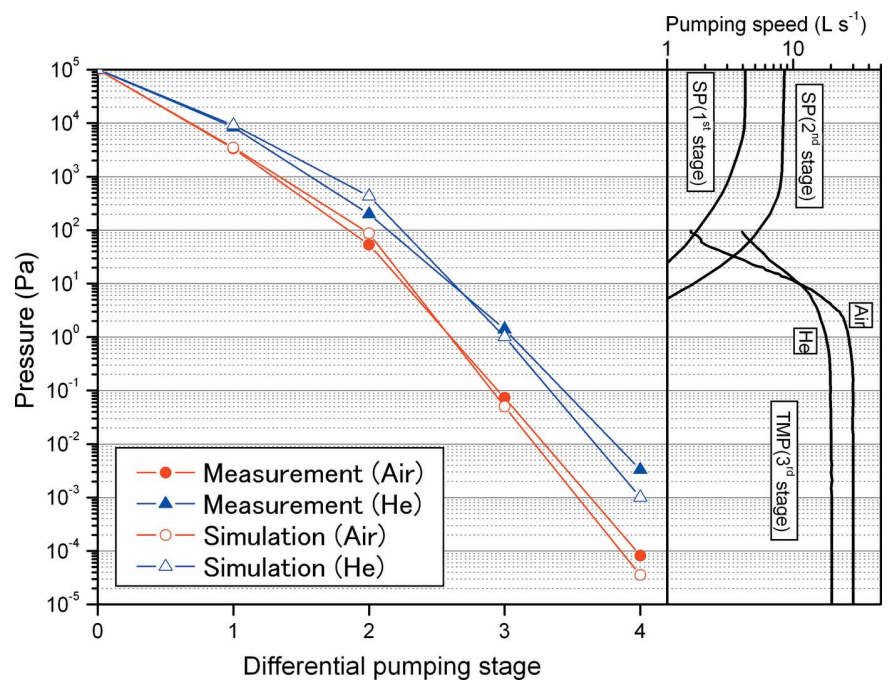

Figure 4

(Left) Performance measurement of the present differential pumping system. The pressure distribution curves were measured for the atmospheric pressure of air (filled circles) and helium (filled triangles) conditions. The calculated pressure for the optimized design is also indicated for air (empty circles) and helium (empty triangles). (Right) Pumping speed curves of the vacuum pumps used in the present system. 
ering the conductance of the piping length, which was estimated for a $1 \mathrm{~m}$ straight pipe.

It was necessary to choose vacuum pumps that exhibit a minimum pressure with the designed pumping speed for the system. The most critical region is a pressure range from $10^{-1}$ to $10^{2} \mathrm{~Pa}$. This so-called 'pumping speed gap' is generated between the lower pressure limit of the rough pump and the higher pressure limit of the turbomolecular pump (Gog et al., 2007). In the present system, the pumping speed curves do not show the critical pumping speed gap, indicating that the pressures at all operation points were those in the steady-state operation.

When $P_{0}$ is for atmospheric air, the pressure was reduced to $3.4 \times 10^{3}$ and $4.6 \times 10^{1} \mathrm{~Pa}$ at the first and the second stages, respectively. The pressures at the third and the fourth stages were $1.9 \times 10^{-2}$ and $8.5 \times 10^{-5} \mathrm{~Pa}$, respectively. At each section, the experimentally determined pressure is in agreement with the estimated pressure. The prototype differential pump achieved differential pressures of about nine orders of magnitude less than the atmospheric pressure of air, and it provides excellent isolation between the atmospheric pressure of the sample environment and the high vacuum of the beamline across the short distance.

It was a challenge to connect the experimental chamber filled with $1 \mathrm{~atm}$ of helium gas to the high-vacuum beamline in the windowless system. Fig. 4 shows the results of the performance test and the simulation. The pressure of the $P_{4}$ section increased by a factor of about 40 compared with the $P_{4}$ value for air. The deterioration of the vacuum level for helium is also expected by the simulation. Two possible reasons can be considered. First, the gas flow is more enhanced for a lower- $Z$ gas. For example, as was discussed in $\S 2$, the gas flow $Q$ for helium is 2.6 times larger than that for air in the molecular flow region. Second, turbomolecular pumps have a lower pumping speed for helium compared with air. Although the performance of the system degraded by about one order of magnitude when using helium, the observed $P_{4}$ value $\left(3.3 \times 10^{-3} \mathrm{~Pa}\right)$ is sufficient to connect this system to the beamline.

Stable operation of the prototype differential pumping system was confirmed using atmospheric air in a test that lasted for one month. The stability test using helium lasted for a little over four hours, whose period was limited by the capacity of the helium gas cylinder $\left(7 \mathrm{~m}^{3}\right)$. The system was stable throughout these tests.

The simulation predicts the observed pressures well. The prototype differential pumping system provides excellent isolation between the atmospheric pressure of the sample environment and the high vacuum of the beamline. The validity of the design concept was confirmed, and the system is acceptable for installation on our beamline, even for use with helium.

\subsection{Soft X-ray transmission measurements under the atmospheric helium condition}

The transmission of soft X-ray photons was measured using the soft X-ray photochemistry beamline (BL27SU) at the

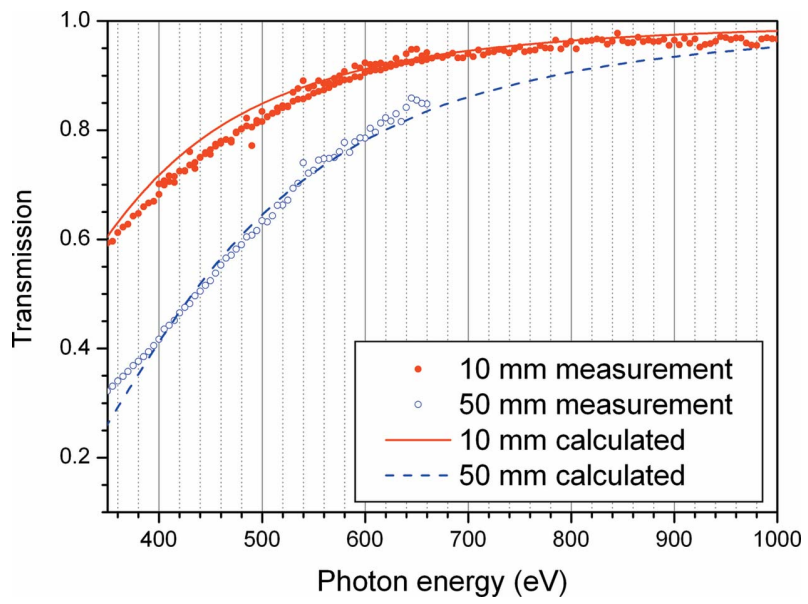

Figure 5

Experimental and calculated transmission curves of soft X-ray photons in the $350 \mathrm{eV}$ to $1000 \mathrm{eV}$ range. The transmission curves were obtained under helium-path conditions. The experimental transmission curves were measured at the helium-path length of $10 \mathrm{~mm}$ (filled circles) and $50 \mathrm{~mm}$ (empty circles). The solid $(10 \mathrm{~mm})$ and dashed $(50 \mathrm{~mm})$ lines are the simulated transmission curves of the fabricated differential pumping system (Henke et al., 1995-2007).

SPring-8 facility (Ohashi et al., 2001a,c; Tamenori et al., 2002). The transmission was determined by measuring $I / I_{0}$ curves. The $I_{0}$ curve was measured under vacuum conditions, where a four-way cross chamber was attached to the end of a fabricated differential pumping system and the chamber was evacuated by a scroll pump. Under these pressure conditions the $P_{0}$ pressure was $4.5 \times 10^{-1} \mathrm{~Pa}$ and the decrease in photon flux caused by the absorption and the scattering of residual gas was less than 1\% (Henke et al., 1995-2007). The $I$ curve was measured, where the $P_{0}$ region was filled with atmospheric helium. The photon intensity was measured by using a $\mathrm{Si}$ photodiode detector (IRD: AXUV-100) that was installed in the four-way cross chamber.

Fig. 5 shows the transmission curves of soft X-rays under atmospheric helium. The distance between the first aperture and the photodiode detector could be changed from $10 \mathrm{~mm}$ to $50 \mathrm{~mm}$. The pressures used in the simulations were the experimentally determined values for the fabricated system. The experimentally determined transmission curves are almost identical to the simulated ones. The transmission at $550 \mathrm{eV}$ (the oxygen $K$-edge region) measured at $10 \mathrm{~mm}$ downstream of the first aperture is about $85 \%$. When the photon flight path in the atmospheric pressure region is expanded to $50 \mathrm{~mm}$, more than $70 \%$ of the incident photons are transmitted to the detector. The soft X-ray photons decrease by about $15 \%$ while passing through the extra $40 \mathrm{~mm}$ of the atmospheric-pressure region. The transmission decreased in the lower photon energy region and the transmission was reduced to $60 \%$ at $350 \mathrm{eV}$. The prototype differential pump was a success: low-energy soft X-ray photons passed through the atmospheric pressure environment.

The red curve in Fig. 6 indicates the simulated transmission of the prototype windowless differential pumping system with the helium path, that is, the transmission in the sample region was omitted from the simulated transmission curve shown in 


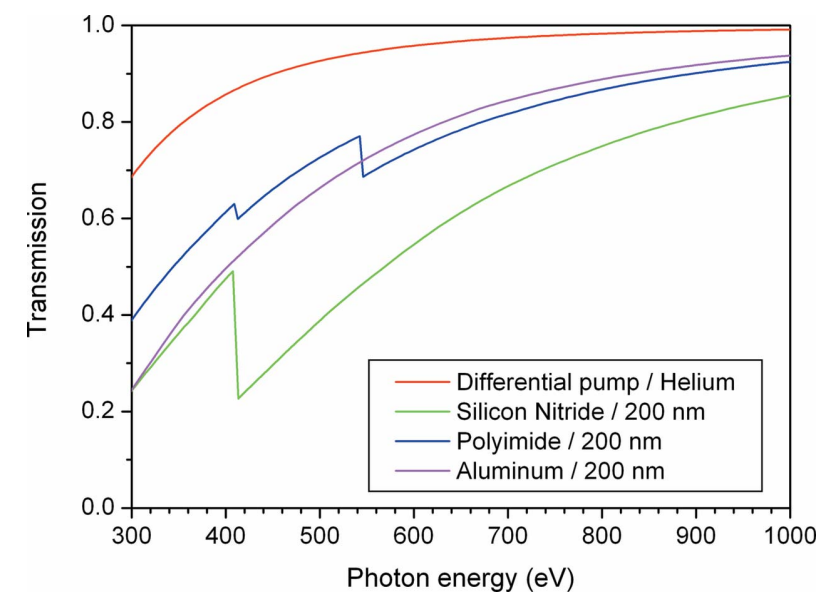

Figure 6

Calculated transmission curves of soft X-ray photons through the windowless differential pump using the helium path compared with various vacuum windows used in the soft X-ray region.

Fig. 5. Fig. 6 indicates that the significant reduction of photon intensity does not occur in the differential pumping sections but in the sample chamber filled by the atmospheric helium. The reduction of the photons in the differential pump section is less than $10 \%$ for soft $\mathrm{X}$-rays above $500 \mathrm{eV}$ and $30 \%$ at $300 \mathrm{eV}$. For comparison, the transmission curves of several vacuum windows used in the soft X-ray region are also indicated in Fig. 6 (Henke et al., 1995-2007). The transmission levels of the prototype differential pump are higher than those using vacuum windows. Additionally, with the windowless differential pump, there are no edge structures resulting from the absorption by the elements contained in the window. The present study demonstrates that the windowless pump allows soft X-rays in a wider energy range at very high efficiencies, compared with conventional vacuum windows.

\section{Conclusion}

A differential pumping system for the application of windowless soft X-ray experiments has been developed. The goal of the design concept was to construct a compact system with no degradation in the performance of the differential pump. The steady-state pressures of the differential pump were simulated by use of simple formulas for vacuum. The prototype system was assembled to confirm the validity of the design concept. The assembled system was an aperture-based compact design, and the total length of the system was less than $600 \mathrm{~mm}$. The simulated pressures were compared with the experimentally measured values. The prototype differential pump achieved differential pressures of about nine orders of magnitude less than the atmospheric pressure of air, and the pressure at the upstream stage was $8.5 \times 10^{-5} \mathrm{~Pa}$. For atmospheric helium, a differential pressure of eight orders of magnitude was achieved, and the pressure at the upstream stage was $3.3 \times 10^{-3} \mathrm{~Pa}$. The simulation used simple formulas to calculate vacuum and predicted the observed pressures well. The prototype differential pumping system provides excellent isolation between the atmospheric pressure of the sample environment and the high vacuum of the beamline.

The differential pumping system was installed on the beamline BL27SU of SPring-8, and the transmission of soft $\mathrm{X}$-rays through the atmospheric helium was measured as a demonstration. The transmission level of the photons in the differential pump section was more than $90 \%$ for soft X-rays above $500 \mathrm{eV}$ and $70 \%$ at $300 \mathrm{eV}$. The observed transmission qualitatively agreed with the simulated results. Transmission of the prototype differential pump was confirmed to be higher than that of various vacuum windows currently used with soft X-rays.

This work was financially supported by a GIGNO project at SPring-8. The author gratefully acknowledges Professor M. Takata for the opportunity that made this work possible and for advice during the review process. The author would also like to thank Mr M. Higashiyama for making some of the key equipment used in this study. Thanks are due to Professor K. Tanaka for careful reading of this manuscript and for useful comments. This study was conducted with the approval of the SPring-8 Proposal Review Committee (Proposal 2007A0355NS1-np, 2008A2061).

\section{References}

Blumer, H., Zelenika, S., Ulrich, J., Betemps, R., Schulz, L., Pfeiffer, F., Flechsig, U., Ellenberger, Urs. \& Wild, C. (2006). Proceedings of the International workshop on Mechanical Engineering Design of Synchrotron Radiation Equipment and Instrumentation 2006, http:// medsi2006.spring8.or.jp/Proc/53.pdf.

Clausing, P. (1932). Ann. Phys. 12, 961; English translation: J. Vac. Sci. Technol. (1971), 8, 636-646.

Gog, T., Casa, D. M., Kuzmenko, I., Krakora, R. J. \& Bolin, T. B. (2007). J. Synchrotron Rad. 14, 339-344.

Goto, S., Yabashi, M., Tamasaku, K., Takahashi, S. \& Ishikawa, T. (2004). Proceedings of the 8th International Conference on Synchrotron Radiation Instrumentation, pp. 400-403. San Fransisco, USA.

Haberland, H. (1994). Editor. Clusters of Atoms and Molecules. Berlin: Springer-Verlag.

Henke, B. L., Gullikson, E. M. \& Davis, J. C. (1995-2007). X-ray Optics Tools, http://www-cxro.lbl.gov/.

Lee, J. C., Ueng, T. S., Chen, J. R., Hsu, Y. J., Hsiung, G. Y., Lin, T. F., Chang, S. H., Hsu, S. N. \& Wang, D. J. (2001). Nucl. Instrum. Methods Phys. Res. A, 467-468, 793-796.

Mercier, B., Compin, M., Prevost, C., Bellec, G., Thissen, R., Dutuit, O. \& Nahon, L. (2000). J. Vac. Sci. Technol. A, 18, 2533-2541.

Ogletree, D. F., Bluhm, H., Lebedev, G., Fadley, C. S., Hussain, Z. \& Salmeron, M. (2002). Rev. Sci. Instrum. 73, 3872-3877.

O'Hanlon, J. F. (2003). A User's Guide to Vacuum Technology, 3rd ed. Hoboken: John Wiley and Sons.

Ohashi, H., Goto, S., Kishimoto, H., Murase, T., Isshiki, M., Suzuki, Y., Kimura, H., Takeshita, K. \& Ishikawa, T. (2001b). Nucl. Instrum. Methods Phys. Res. A, 467, 801-804.

Ohashi, H., Ishiguro, E., Tamenori, Y., Kishimoto, H., Tanaka, M., Irie, M., Tanaka, T. \& Ishikawa, T. (2001a). Nucl. Instrum. Methods Phys. Res. A, 467, 529-532.

Ohashi, H. et al. (2001c). Nucl. Instrum. Methods Phys. Res. A, 467, 533-536.

Pantförder, J., Pöllmann, S., Zhu, J. F., Borgmann, D., Denecke, R. \& Steinrück, H.-P. (2005). Rev. Sci. Instrum. 76, 014102. 
Pollard, J. E., Trevor, D. J., Lee, Y. T. \& Shirey, D. A. (1981). Rev. Sci. Instrum. 52, 1837-1846.

Ran, Q., Matsiev, D., Wodtke, A. M. \& Auerbach, D. J. (2007). Rev. Sci. Instrum. 78, 104104.

Renier, M. \& Draperi, A. (1997). Vacuum, 48, 405-407.

Roper, M. D., Van de Laan, G., Flaherty, J. V. \& Padmore, H. A. (1992). Rev. Sci. Instrum. 63, 1482-1485.

Rowe, J. E., Christman, S. B. \& Chaban, E. E. (1973). Rev. Sci. Instrum. 44, 1675-1676.

Rowe, J. E., Christman, S. B. \& Chaban, E. E. (1985). Rev. Sci. Instrum. 56, 759-761.
Rozanov, L. N. \& Hablanian, M. H. (2002). Editor. Vacuum Technique. New York: Taylor and Francis. Santeler, D. J. (1986a). J. Vac. Sci. Technol. A, 4, 338-343.

Santeler, D. J. (1986b). J. Vac. Sci. Technol. A, 4, 348-352.

Santeler, D. J. (1994). J. Vac. Sci. Technol. A, 12, 1744-1749.

Tamenori, Y., Ohashi, H., Ishiguro, E. \& Ishikawa, T. (2002). Rev. Sci. Instrum. 73, 1588.

Warburton, W. K. \& Pianetta, P. (1990). Nucl. Instrum. Methods Phys. Res. A, 291, 350-356.

Yagi, S., Matsumura, Y., Soda, K., Hashimoto, E. \& Taniguchi, M. (2004). Surf. Interface Anal. 36, 1064-1066. 